\title{
Simple Synthesis and Characterization of Cobalt Ferrite Nanoparticles by a Thermal Treatment Method
}

\author{
Mahmoud Goodarz Naseri, ${ }^{1,2}$ Elias B. Saion, ${ }^{1}$ Hossein Abbastabar Ahangar, ${ }^{3}$ \\ Abdul Halim Shaari, ${ }^{1}$ and Mansor Hashim ${ }^{1,4}$ \\ ${ }^{1}$ Department of Physics, Putra University, Malaysia (UPM), 43400 Serdang, Selangor, Malaysia \\ ${ }^{2}$ Department of Physics, Faculty of Science, Malayer University, Malayer, Iran \\ ${ }^{3}$ Department of Chemistry, Putra University, Malaysia (UPM), 43400 Serdang, Selangor, Malaysia \\ ${ }^{4}$ Advanced Materials and Nanotechnology Laboratory, Institute of Advanced Technology, Putra University, Malaysia (UPM), \\ 43400 Serdang, Selangor, Malaysia
}

Correspondence should be addressed to Mahmoud Goodarz Naseri, mahmoud.naseri55@gmail.com

Received 4 July 2010; Revised 20 November 2010; Accepted 26 November 2010

Academic Editor: Michael Harris

Copyright (C) 2010 Mahmoud Goodarz Naseri et al. This is an open access article distributed under the Creative Commons Attribution License, which permits unrestricted use, distribution, and reproduction in any medium, provided the original work is properly cited.

Crystalline, magnetic, cobalt ferrite nanoparticles were synthesized from an aqueous solution containing metal nitrates and polyvinyl pyrrolidone (PVP) as a capping agent by a thermal treatment followed by calcination at various temperatures from 673 to $923 \mathrm{~K}$. The structural characteristics of the calcined samples were determined by X-ray diffraction (XRD), Fourier transform infrared spectroscopy (FT-IR), and transmission electron microscopy (TEM). A completed crystallization occurred at 823 and $923 \mathrm{~K}$, as shown by the absence of organic absorption bands in the FT-IR spectrum. Magnetization measurements were obtained at room temperature by using a vibrating sample magnetometer (VSM), which showed that the calcined samples exhibited typical magnetic behaviors.

\section{Introduction}

The spinel ferrite structure with the formula of $\mathrm{MFe}_{2} \mathrm{O}_{4}$ ( $\mathrm{M}$ $=\mathrm{Co}, \mathrm{Ni}, \mathrm{Zn}$, or other metals) can be described as a cubic, closely packed arrangement of oxygen atoms, and $\mathrm{M}^{2+}$ and $\mathrm{Fe}^{3+}$ ions can occupy either tetrahedral (A) or octahedral (B) sites [1]. Spinel ferrite nanoparticles have attracted much attention because of their electronic, magnetic, and catalytic properties, all of which are different from those of their bulk counterparts. Among spinel ferrites, cobalt ferrite $\left(\mathrm{CoFe}_{2} \mathrm{O}_{4}\right)$ has an inverse spinel structure in which, in the ideal state, all $\mathrm{Co}^{2+}$ ions are in $\mathrm{B}$ sites, and $\mathrm{Fe}^{3+}$ ions are equally distributed between A and B sites. Cobalt ferrite has been widely studied due to its high electromagnetic performance, excellent chemical stability, mechanical hardness, high coercivity, and moderate saturation magnetization, which make it a good candidate for the electronic components used in computers, recording devices, and magnetic cards [2-4].
These properties are dependent on chemical composition and microstructural characteristics, which can be controlled in the fabrication and synthesis processes.

In order to acquire materials with the desired physical and chemical properties, the preparation of cobalt ferrite nanoparticles through different routes has become an important area of research and development. Various methods of synthesizing spinel cobalt ferrite nanoparticles have been reported, such as ball milling [5], a ceramic method by firing [5], coprecipitation [5-7], reverse micelles [8], hydrothermal methods $[9,10]$, a polymeric precursor [11], sol-gel [12], microemulsions [13], laser ablation [14], a polyol method [15], sonochemical approaches [16], and aerosol method [17]. Various precipitation agents have been used to prepare cobalt ferrite nanoparticles of a specific size and shape, for example, metal hydroxide in the coprecipitation method, surfactant and ammonia in the reverse micelles and microemulsion methods, and organic matrices 
in the polymeric precursor, sol-gel, and polyol methods. Most of these methods have achieved nanoparticles of the required sizes and microstructures, but they are difficult to apply on larger scales because of their expensive and complicated procedures, high reaction temperatures, long reaction times, toxic reagents and by-products, and their potential harm to the environment.

In the present study, cobalt ferrite nanoparticles were synthesized from an aqueous solution containing metal nitrates, polyvinyl pyrrolidone, and deionized water using a thermal treatment method followed by crushing and calcination. This method does not require the addition of any other chemicals to the solution, and it has the advantages of simplicity, a low cost, a lack of by-product effluents, and an environmentally friendly operation. The textural and morphological characteristics of the prepared cobalt ferrite nanoparticles were studied with various techniques to verify the particle size and distribution as well as to explore other parameters of interest.

\section{Experimental}

2.1. Materials. Metal nitrate reagents were used as precursors, poly(vinyl pyrrolidone) (PVP) was used as a capping agent to reduce the agglomeration of the particles, and deionized water was used as the solvent. Iron nitrate, $\mathrm{Fe}\left(\mathrm{NO}_{3}\right)_{3} \cdot 9 \mathrm{H}_{2} \mathrm{O}$, and cobalt nitrate, $\mathrm{Co}\left(\mathrm{NO}_{3}\right)_{2} \cdot 6 \mathrm{H}_{2} \mathrm{O}$, were purchased from Acros Organics with purities exceeding 99\%. PVP $(M W=29000)$ was purchased from Sigma Aldrich and was used without further purification. An aqueous solution of PVP was prepared by dissolving $3 \mathrm{~g}$ of polymer in $100 \mathrm{ml}$ of deionized water at $343 \mathrm{~K}$, before mixing $0.2 \mathrm{mmol}$ of iron nitrate and $0.1 \mathrm{mmol}$ of cobalt nitrate $(\mathrm{Fe}: \mathrm{Co}=2: 1)$ into the polymer solution and constantly stirring for $2 \mathrm{~h}$ using a magnetic stirrer until a colorless, transparent solution was obtained. The solution had a $\mathrm{pH}$ from 1 to 2 measured by means of a glass electrode. No precipitation of materials occurred before the heat treatment. The mixed solution was poured into a glass Petri dish and heated at $353 \mathrm{~K}$ in an oven for $24 \mathrm{~h}$ to evaporate the water. The resulting orange solid was crushed for $15 \mathrm{~min}$ in a mortar to form powder. The calcinations of the powder was conducted at 673,723 , 823 , and $923 \mathrm{~K}$ for $3 \mathrm{~h}$ for the decomposition of the organic compounds and the crystallization of the nanoparticles.

2.2. Characterization. The characterization of the prepared cobalt ferrite nanoparticles was conducted by using various techniques to verify the particle size and distribution and to explore other parameters of interest. The structure of the $\mathrm{CoFe}_{2} \mathrm{O}_{4}$ nanoparticles was characterized by the XRD technique using a Shimadzu diffractometer (model XRD 6000) using $\mathrm{Cu} \mathrm{K} \mathrm{K}_{\alpha}(0.154 \mathrm{~nm})$ radiation to generate diffraction patterns from the crystalline powder samples at ambient temperature over the $2 \theta$ range from $10^{\circ}$ to $80^{\circ}$. Infrared spectra $\left(280-4000 \mathrm{~cm}^{-1}\right)$ were recorded using an FT-IR spectrometer (Perkin Elmer model 1650), with the samples pressed onto diamond-coated CsI pellets. Both the XRD and the FT-IR results were used to establish the crystallinity of the prepared cobalt ferrite powder at different calcination temperatures. The structural characteristics and particle size of the nanocrystalline powder were determined at room temperature by transmission electron micrograph (TEM) (JEOL 2010F UHR version electron microscope) operating at an accelerating voltage of $200 \mathrm{kV}$. Selected areas were obtained on the TEM images to ascertain both morphology and particle size distribution at different calcination temperatures. Magnetic characterization of the cobalt ferrite nanoparticles was performed by using a vibrating sample magnetometer (VSM) (Lake Shore 4700) at room temperature with a maximum magnetic field of $15 \mathrm{kOe}$.

\section{Results and Discussion}

The interactions between PVP and metal ions are represented schematically in Figure 1, which shows that the cobalt (II) and iron (III) ions are bound by the strong ionic bonds between the metallic ions and the amide group in a polymeric chain or between the polymeric chains.

This uniform immobilization of metallic ions in the cavities of the polymer chains favors the formation of a uniformly distributed, solid solution of the metallic oxides in the calcination process.

Figure 2 shows the XRD patterns of the different samples. A broad peak is revealed in the precursor (Figure 2(a)), which does not have sharp diffraction patterns and is still amorphous. The calcined samples at $673,723,823$, and $923 \mathrm{~K}$ (ICDD PDF: 22-1086) show the reflection planes of (111), (220), (222), (311), (400), (422), (511), (440), and (533), which confirms the presence of cobalt ferrite with a face-centered cubic structure [18]. Our results show that, as the calcination temperature increases, the diffraction peaks become sharper and narrower, and their intensity increases. This indicates an intensification in crystallinity that originates from the increment of crystalline volume ratio due to the particle size enlargement of the nuclei [19]. The impure phase of $\alpha-\mathrm{Fe}_{2} \mathrm{O}_{3}$ is found in all calcined samples. This impurity occurs naturally as hematite [20].

The average crystallite size of calcined sample obtained at $923 \mathrm{~K}$, for five planes with higher intensities that consist of (311), (440), (220), (511), and (400), was estimated as $8.357 \pm 0.003 \mathrm{~A}^{\circ}$, which is in relatively good agreement with the bulk value of $8.377 \mathrm{~A}^{\circ}[21]$. The average particle size also was determined from the full width at the half maximum (FWHM) of the XRD patterns, using the well-known Scherer formula:

$$
D=0.9 \frac{\lambda}{\beta} \cos \theta
$$

where $\mathrm{D}$ is the crystallite size $(\mathrm{nm}), \beta$ is the full width of the diffraction line at half of the maximum intensity measured in radians, $\lambda$ is the X-ray wavelength of $\mathrm{Cu} \mathrm{K}_{\alpha}=0.154 \mathrm{~nm}$, and $\theta$ is the Bragg angle [22]. The particle sizes estimated using the Scherer formula were found to increase with the calcination temperature, from about $10 \mathrm{~nm}$ at $673 \mathrm{~K}$ to about $41 \mathrm{~nm}$ at $923 \mathrm{~K}$, as shown in Table 1.

Figure 3 shows the FT-IR spectra analysis with wave numbers between 280 and $4000 \mathrm{~cm}^{-1}$ for precursor and 
TABLE 1: Average particle size (nm) of $\mathrm{CoFe}_{2} \mathrm{O}_{4}$ nanoparticles determined from XRD and TEM and magnetic properties observed from VSM technique at room temperature for calcined samples at $673,723,823$, and $923 \mathrm{~K}$.

\begin{tabular}{lccccccc}
\hline $\begin{array}{l}\mathrm{CoFe}_{2} \mathrm{O}_{4} \\
\text { nanoparticles }\end{array}$ & $\begin{array}{c}\text { Calcination } \\
\text { temperature } \\
(\mathrm{K})\end{array}$ & $\begin{array}{c}\text { Average } \\
\text { particle size } \\
\text { XRD }(\mathrm{nm})\end{array}$ & $\begin{array}{c}\text { Average } \\
\text { particle size } \\
\text { TEM }(\mathrm{nm})\end{array}$ & $\begin{array}{c}\text { Saturation } \\
\text { magnetization } \\
M_{s}(\mathrm{emu} / \mathrm{g})\end{array}$ & $\begin{array}{c}\text { Remanent } \\
\text { magnetization } \\
M_{r}(\mathrm{emu} / \mathrm{g})\end{array}$ & $\begin{array}{c}\text { Coercivity } \\
H_{c}(\mathrm{Oe})\end{array}$ & $\begin{array}{c}\text { Remanence } \\
\text { ratio }\left(M_{r} / M_{s}\right)\end{array}$ \\
\hline CoFerrite 1 & 673 & 10 & 12.5 & 1.15 & 0.10 & 138 & 0.087 \\
CoFerrite 2 & 723 & 15.5 & 14 & 2.14 & 0.57 & 823 & 0.266 \\
CoFerrite 3 & 823 & 34 & 29 & 18.02 & 9.02 & 1791 & 0.500 \\
CoFerrite 4 & 923 & 41 & 39 & 28.52 & 11.61 & 1002 \\
\hline
\end{tabular}

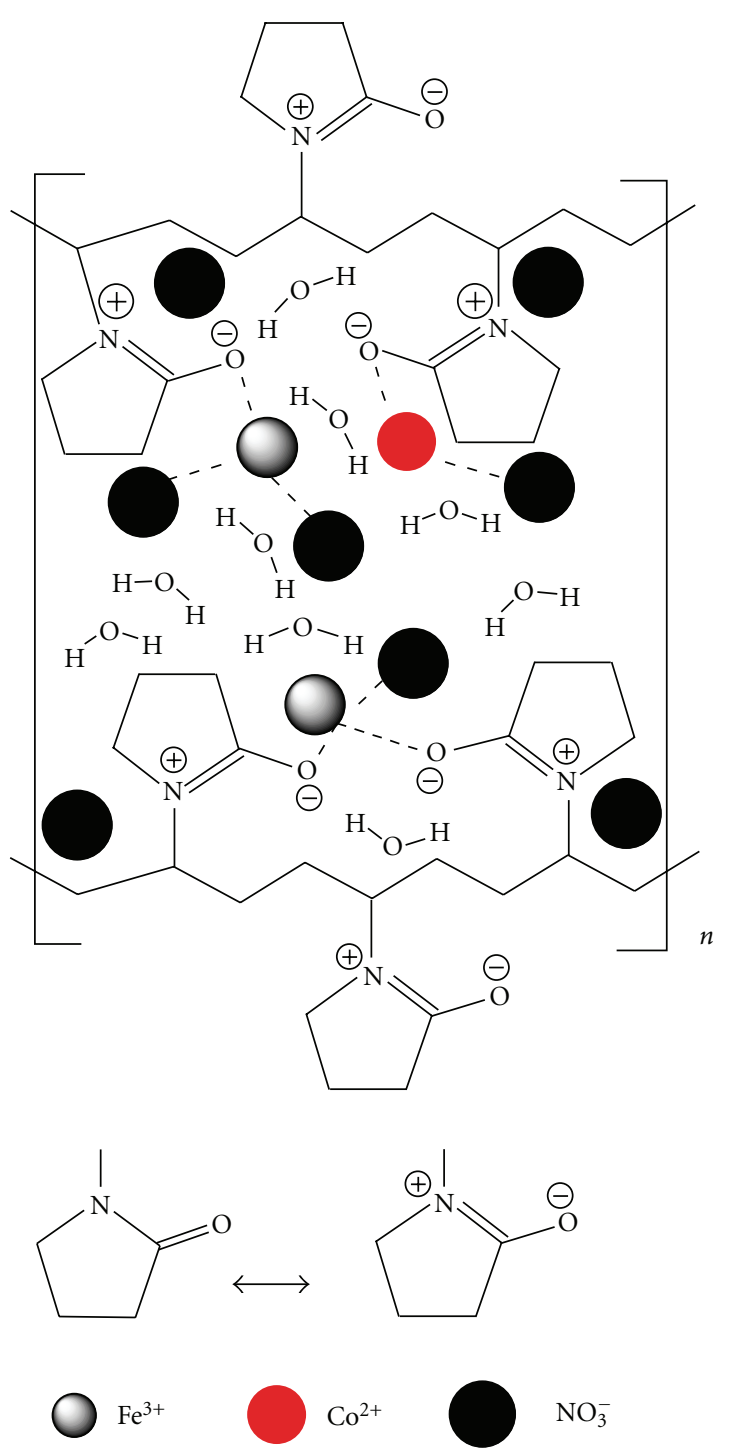

FIGURE 1: A proposed mechanism of interactions between PVP and metal ions before calcination.

calcined samples. Figure 3(a) shows all absorption peaks in the precursor, which were attributed to PVP. The most important peaks are at 3345, 1638, 1430, 1230, 842, and $670 \mathrm{~cm}^{-1}$, corresponding to the stretching and bending vibrations of $\mathrm{O}-\mathrm{H}, \mathrm{C}=\mathrm{O}, \mathrm{H}-\mathrm{C}-\mathrm{H}, \mathrm{C}-\mathrm{H}, \mathrm{C}-\mathrm{C}$, and $\mathrm{C}-\mathrm{N}=\mathrm{O}$, respectively [23]. It must be mentioned that the absorption

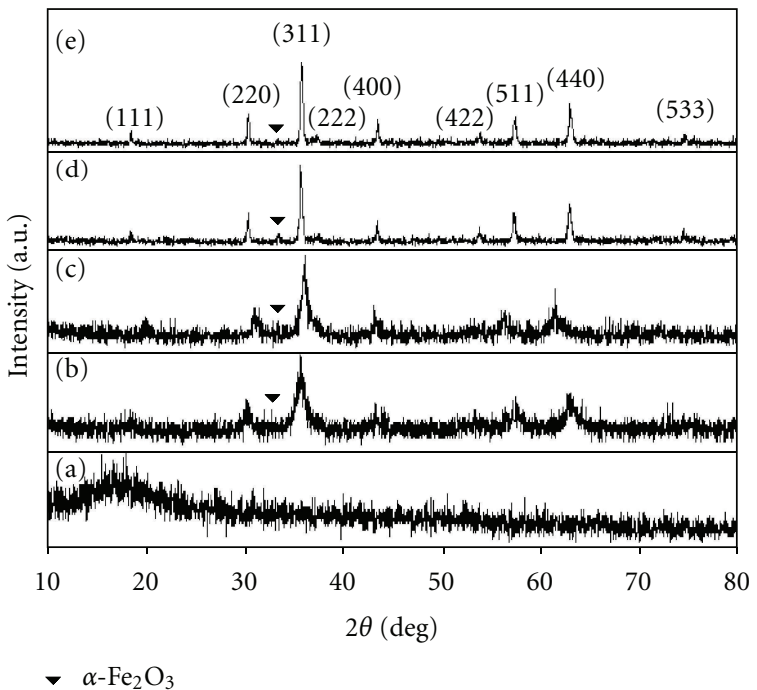

FIGURE 2: XRD patterns of (a) precursor and cobalt ferrite nanoparticles calcined at (b) 673, (c) 723, (d) 823, and (e) $923 \mathrm{~K}$.

peaks of the nitrate anion overlap with vibration of $\mathrm{C}-\mathrm{H}$ at 1230 and $1430 \mathrm{~cm}^{-1}$.

The cobalt ferrite samples that had been prepared were further studied for the interaction between the formation of the crystalline $\mathrm{CoFe}_{2} \mathrm{O}_{4}$ nanostructure and the PVP matrix at different calcination temperatures. The vibrational spectra of the absorption bands of pure spinel cobalt ferrite nanoparticles calcined at $823 \mathrm{~K}$ were observed at 334 and $545 \mathrm{~cm}^{-1}$, whereas the spectra for the calcined samples at $923 \mathrm{~K}$ were observed at 331 and $540 \mathrm{~cm}^{-1}$ (Figures 3(d) and $3(\mathrm{e}))$. This indicates the formation of the spinel $\mathrm{CoFe}_{2} \mathrm{O}_{4}$ nanostructure, as suggested by previously published data $[6,24]$. The FT-IR absorption bands of solids are usually assigned to the vibration of ions in the crystal lattice [25]. The absence of the peaks at $1000-1300 \mathrm{~cm}^{-1}$ and $2000-$ $3000 \mathrm{~cm}^{-1}$ in the samples at $823 \mathrm{~K}$, and higher, confirmed the nonexistence of the $\mathrm{O}-\mathrm{H}$ mode, $\mathrm{C}-\mathrm{O}$ mode, and $\mathrm{C}=\mathrm{H}$ stretching-mode of organic sources [26]. This FT-IR analysis is very useful for establishing the temperature of calcination at 823 and $923 \mathrm{~K}$ by removing the presence of unwanted ions, which may pollute the crystal lattice during preparation. At the lower temperature of $823 \mathrm{~K}$, however, there is still a trace of a broadband absorption peak at $3397 \mathrm{~cm}^{-1}$ due to $\mathrm{O}-\mathrm{H}$ stretching vibration (Figure $3(\mathrm{c})$ ). The absorption 


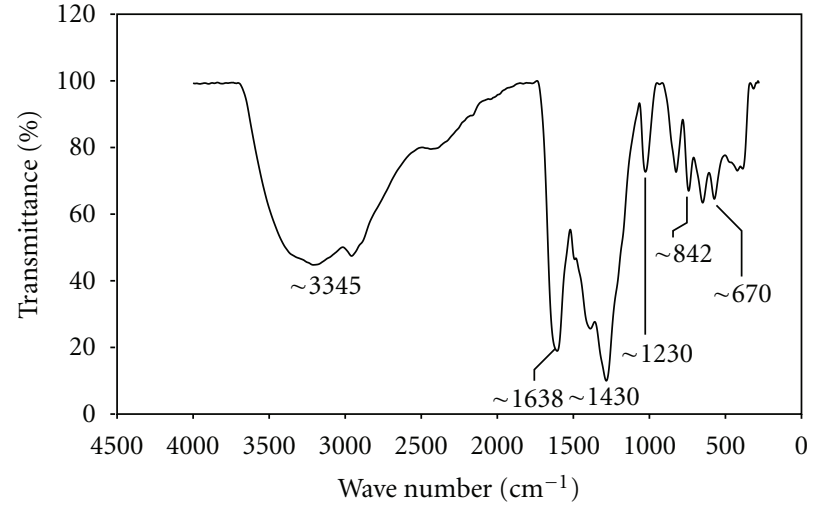

(a)

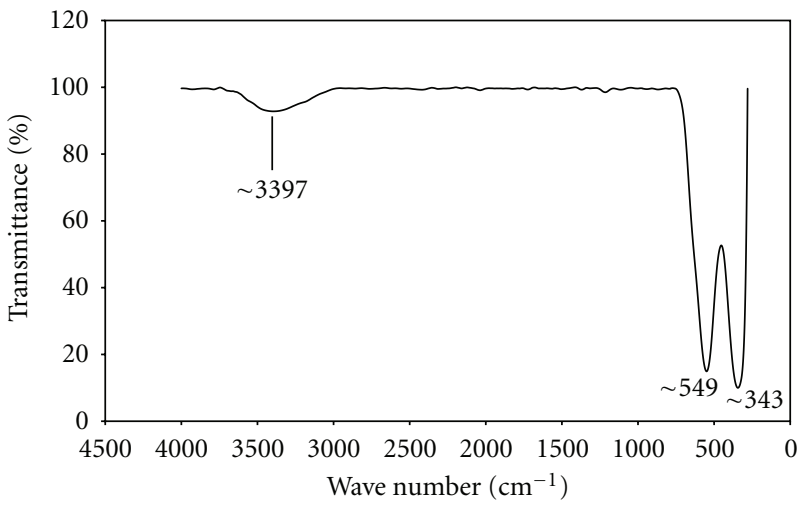

(c)

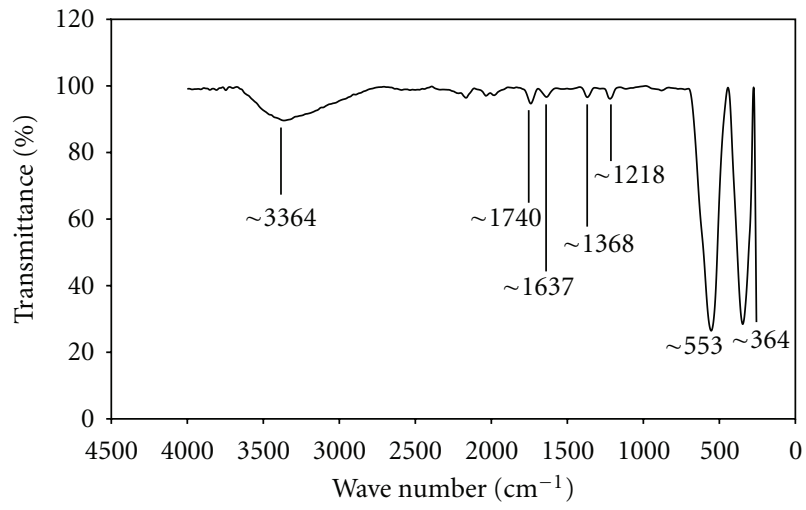

(b)

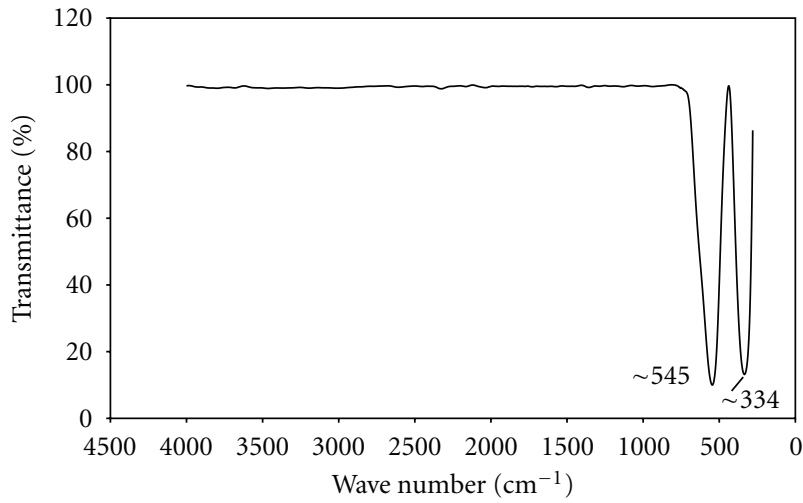

(d)

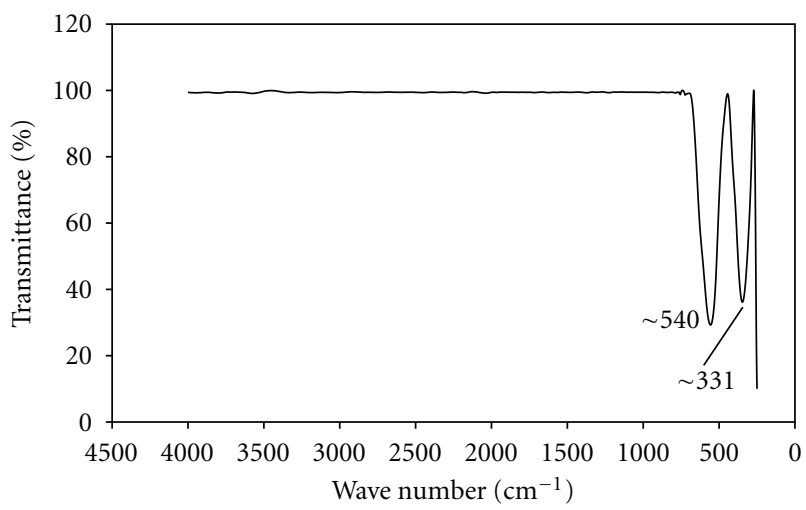

(e)

FIGURE 3: FTIR spectra of (a) precursor and cobalt ferrite nanoparticles calcined at (b) 673, (c) 723, (d) 823 and (e) $923 \mathrm{~K}$ in the range of $280-4000 \mathrm{~cm}^{-1}$.

bands of $\mathrm{Co} \leftrightarrow \mathrm{O}$ and $\mathrm{Fe} \leftrightarrow \mathrm{O}$ bonds appeared at 364 and $553 \mathrm{~cm}^{-1}$, respectively, for the calcined sample at $673 \mathrm{~K}$. As the calcination temperature increased to $923 \mathrm{~K}$, the absorption bands of $\mathrm{Co} \leftrightarrow \mathrm{O}$ and $\mathrm{Fe} \leftrightarrow \mathrm{O}$ bonds shifted to 331 and $540 \mathrm{~cm}^{-1}$, respectively. These bonds are typically associated with pure $\mathrm{CoFe}_{2} \mathrm{O}_{4}$ nanoparticles [6].

The TEM images and particle size distributions of cobalt ferrite samples are shown in Figure 4. The results indicate that the samples prepared by the thermal treatment method are uniform in both morphology and particle size distribution. The particle sizes increased with increasing calcination temperature, which is in good agreement with the XRD results (Table 1). The smallest particle size obtained in this study was $12.5 \mathrm{~nm}$ at $673 \mathrm{~K}$, and particle size reached $39 \mathrm{~nm}$ at the highest calcination temperature of $923 \mathrm{~K}$. This suggests that several neighboring particles fuse together to increase the particle sizes by the melting of their surfaces [27]. All organic materials are removed at $823 \mathrm{~K}$ 

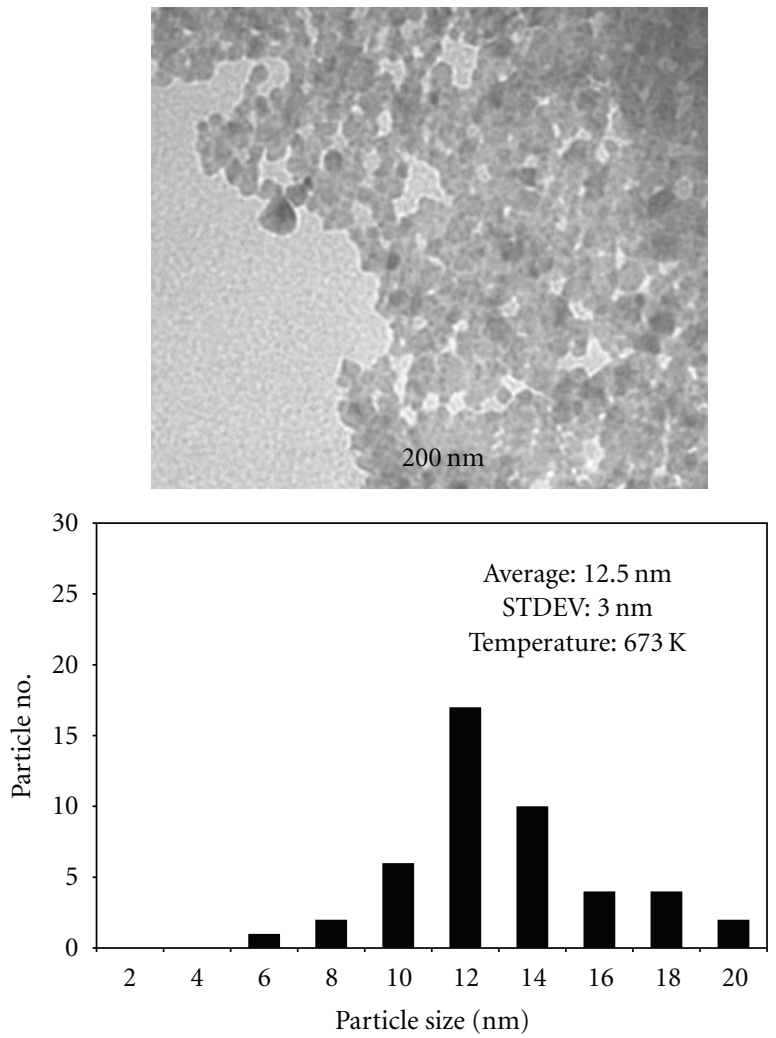

(a)
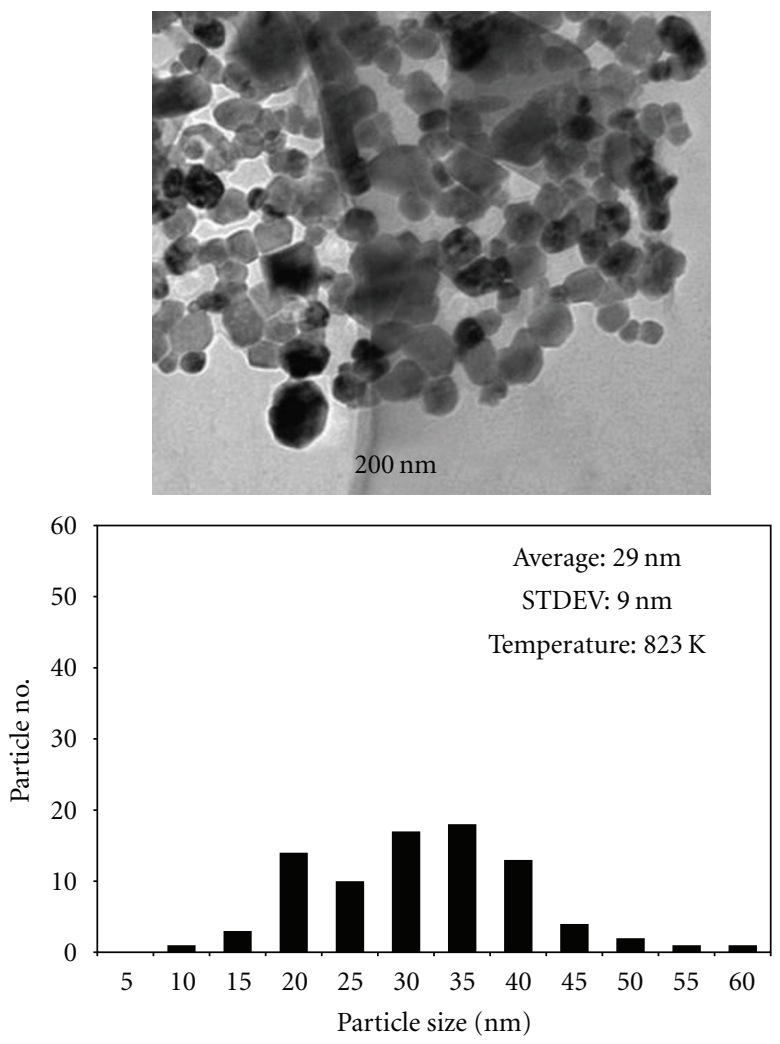

(c)
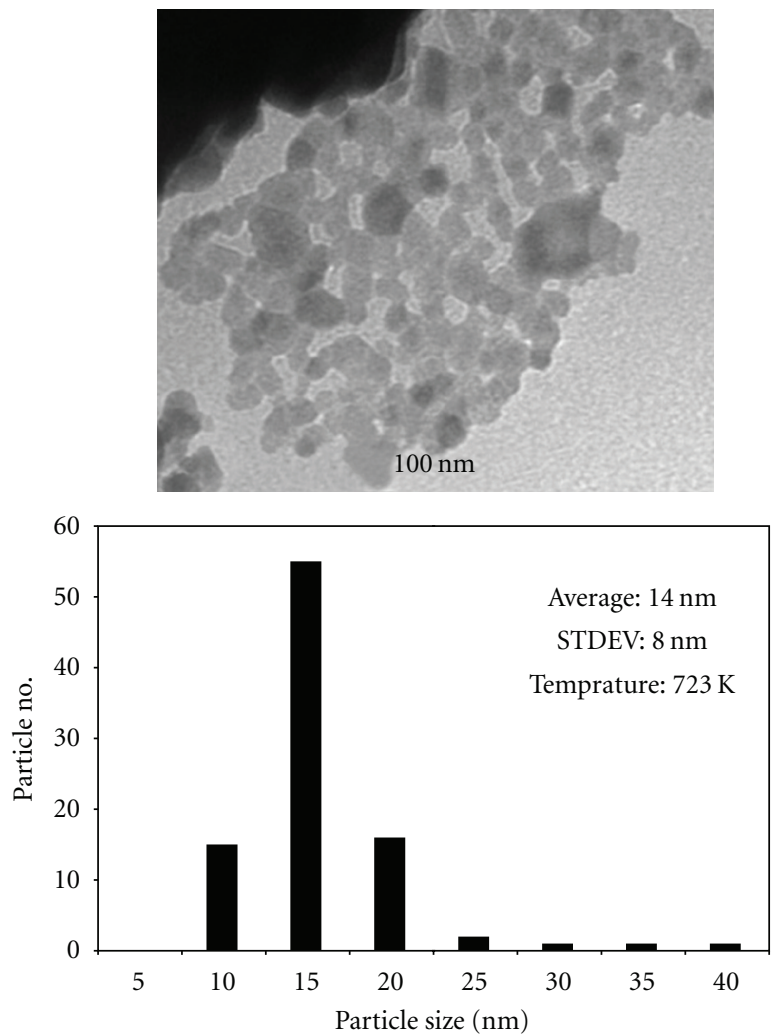

(b)
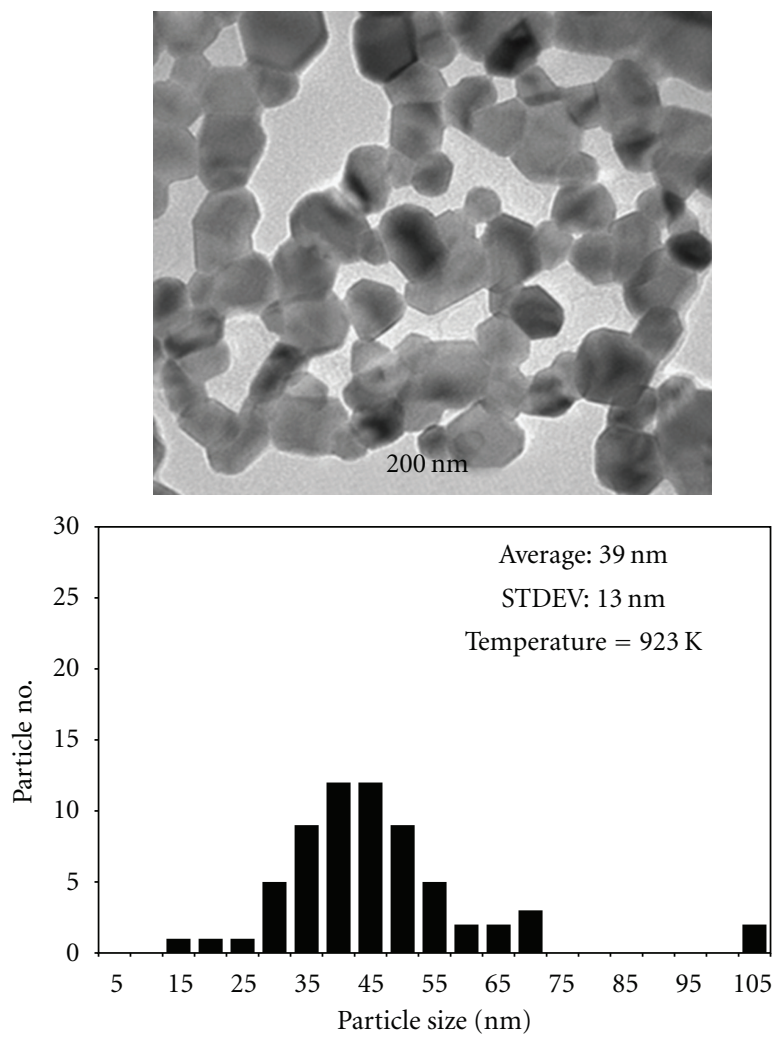

(d)

FIGURE 4: TEM images and particle size distribution histograms of cobalt ferrite nanoparticles at different calcination temperatures: (a) 673, (b) 723, (c) 823 , and (d) $923 \mathrm{~K}$. 


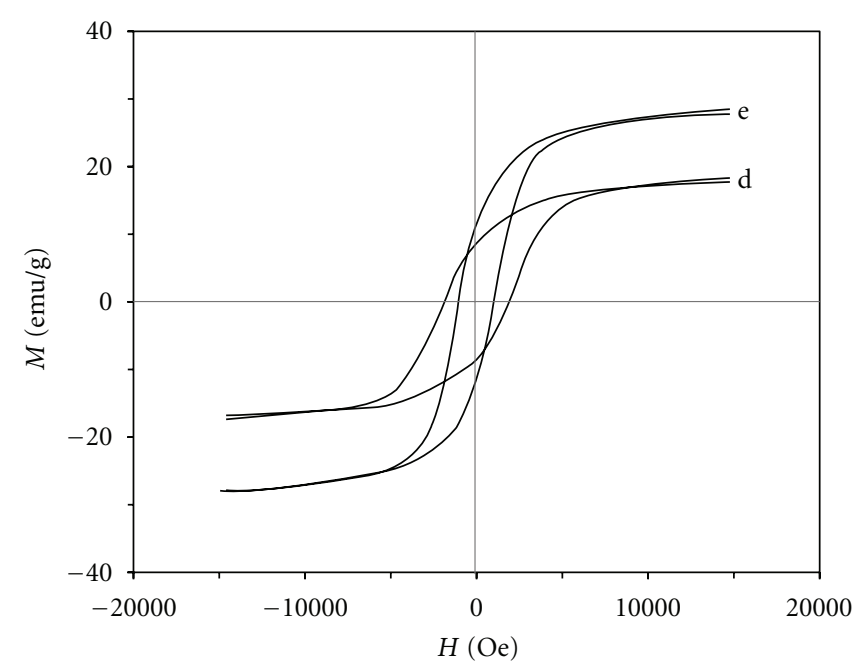

(a)

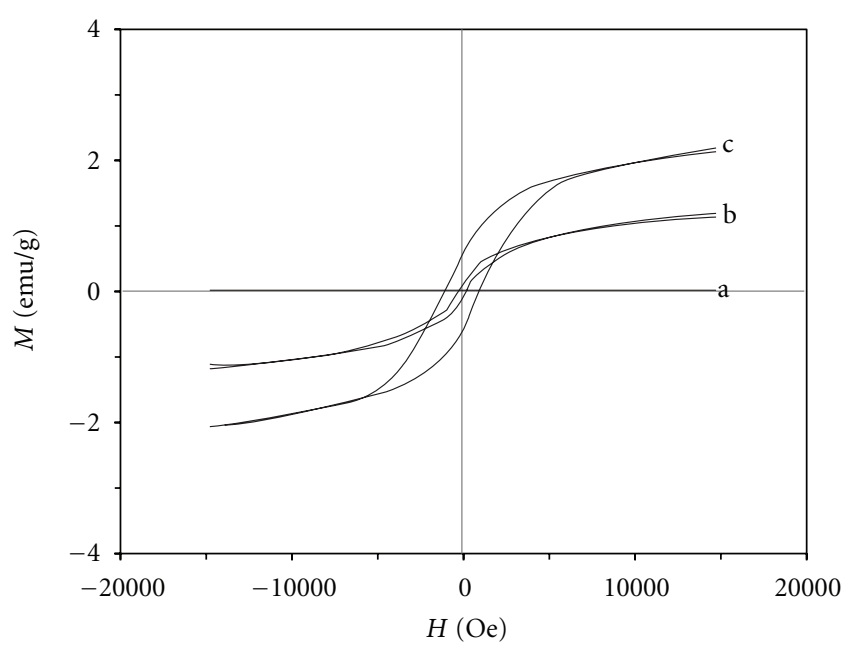

(b)

Figure 5: Magnetization loops of (a) precursor and cobalt ferrite nanoparticles calcined at (b) 673, (c) 723, (d) 823, and (e) 923 K.

(Figure 3(c)), and the particle size also is smaller than that in the sample calcined at $923 \mathrm{~K}$ (Figure $4(\mathrm{c})$ ). This grain growth of particle size enlargement at higher calcination temperatures has previously been observed in zinc ferrite [28] and nickel ferrite [29] systems.

Figure 5 shows the $M(H)$ loops that were measured at room temperature in the range of approximately -15 to $+15 \mathrm{kOe}$. Except for the precursor (Figure 5(a)), which was a nonmagnetic material, the calcined samples exhibited typical magnetic behaviors. It is evident in Table 1 that, when the calcinations temperature increases from 673 to $923 \mathrm{~K}$, the saturation magnetization increases from 1.15 to $28.52 \mathrm{emu} / \mathrm{g}$ at room temperature. The largest saturation magnetization was $28.52 \mathrm{emu} / \mathrm{g}$ for the sample calcined at $923 \mathrm{~K}$, which is much lower than that reported for the multidomain, bulk cobalt ferrite $(74.08 \mathrm{emu} / \mathrm{g})$ [30]. The decrease in saturation magnetization of these samples, compared to that of bulk material, depends on different parameters. In the thermal treatment method, the heating rate of calcination is one of the most important parameters that can effectively increase or decrease the saturation magnetization. In this investigation, the heating rate of calcination was $10 \mathrm{~K} / \mathrm{min}$ for cobalt ferrite nanoparticles calcined at $673,723,823$, and $923 \mathrm{~K}$, which was a relatively high heating rate. Therefore, it is possible that calcination at a slower heating rate would allow the crystallization to be more complete, and the magnetic phase could also increase, resulting in larger saturation magnetization.

Sangmanee and Maensiri [24] showed that saturation magnetization increases from 9.7 to $56.5 \mathrm{emu} / \mathrm{g}$ with decreasing the heating rate of calcination from $20 \mathrm{~K} / \mathrm{min}$ to $5 \mathrm{~K} / \mathrm{min}$ in cobalt ferrite nanostructures calcined at $773 \mathrm{~K}$ and fabricated by electrospinning. Also, the cation site occupancy in $\mathrm{CoFe}_{2} \mathrm{O}_{4}$ nanoparticles is different than in the bulk [31]. In fact, since the nanoparticles are the mixed spinel structure type rather than the inverse spinel structure type (bulk) because of the presence of $\mathrm{Co}^{3+}$ ions and also a cation distribution with cobalt ions on the tetrahedral site [32], the saturation magnetization is reduced [33].

In addition, the appearance of the weakly magnetic, impure phase of hematite (shown in Figure 2) can reduce the saturation magnetization $[20,34]$.The variations of saturation magnetization $\left(M_{s}\right)$ and remanent magnetization $\left(M_{r}\right)$ with particle size for cobalt ferrite nanoparticles are shown in Figure 6. The saturation magnetization and remanent magnetization values of the calcined samples increase with increasing particle size, which may be attributed to the surface effects in these nanoparticles.

The surface of the nanoparticles seems to be composed of some distorted or slanted spins that repel the core spins to align the field direction; consequently, the saturation magnetization and remanent magnetization increase for larger sizes and decrease for smaller sizes [35]. But the values of the coercivity field and remanence ratio $\left(R=M_{r} / M_{s}\right)$ have no similar relationship with saturation magnetization and remanent magnetization, because, when the particle size increases from 12.5 to $39 \mathrm{~nm}$, the coercivity field and remanence ratio increase to $1791 \mathrm{Oe}$ and 0.5 for $29 \mathrm{~nm}$ particle size then decreases to 1002 Oe and 0.407 for $39 \mathrm{~nm}$ particle size at room temperature, respectively (Figure 7).

The existence or absence of the different types of intergrain group exchanges is determined by the amount of $R$ that varies from 0 to 1 [36]. It has been reported that $R<0.5$ is for the particle interact by magnetostatic interaction, while $R=0.5$ is for randomly oriented noninteracting particles that undergo coherent rotations [37, 38]. Finally, $1>R>$ 0.5 confirms the existence of exchange-coupling particles. Therefore, the fact that the values of $\mathrm{R}$ for samples calcined at 673,723 , and $923 \mathrm{~K}$ are lower than 0.5 is attributed to particles that interact by magnetostatic interactions, and the calcined sample at $823 \mathrm{~K}$, with $R=0.5$, has randomlyoriented, non-interacting particles. The variation in the value of the coercivity field and remanence ratio with particle size can be explained on the basis of domain structure, critical 


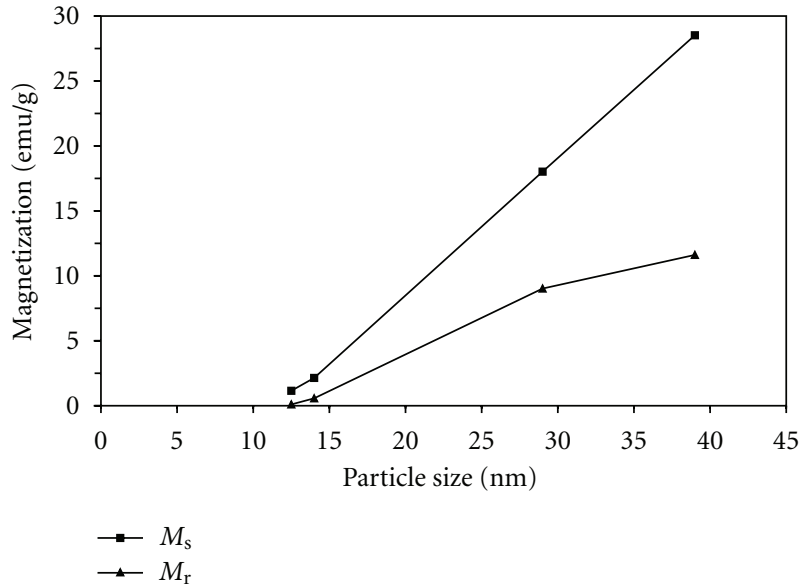

Figure 6: The variation of the saturation magnetization and remanent magnetization with particle size for cobalt ferrite nanoparticles calcined samples at different temperature.

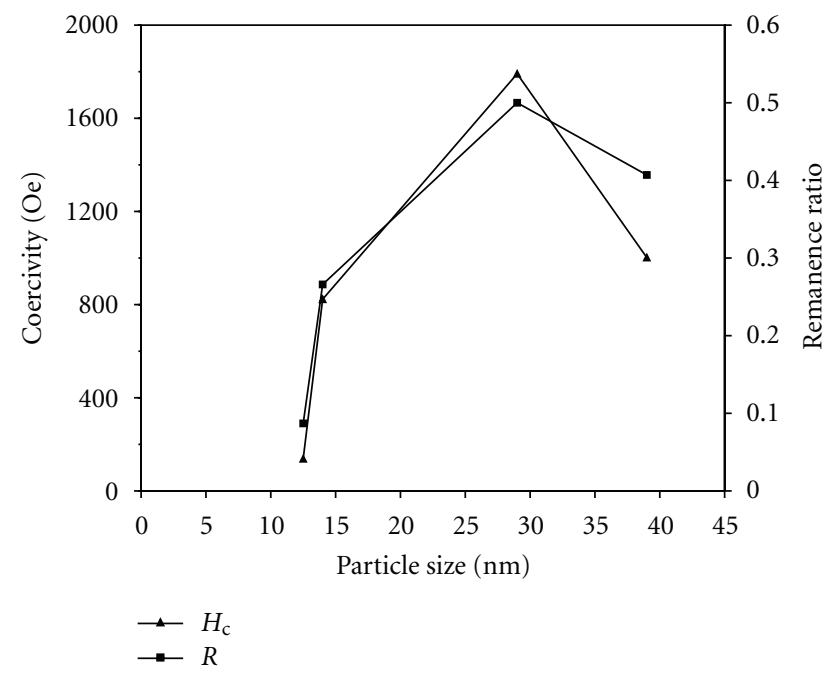

FIGURE 7: The variation of the coercivity field and remanence ratio with particle size for cobalt ferrite nanoparticles calcined samples at different temperature.

size, and the anisotropy of the crystal [27, 39-42]. It is worth mentioning that the magnetic properties of ferrite nanoparticles depend on the preparation method. For instance, 16$\mathrm{nm}$ cobalt ferrite nanoparticles made by co-precipitation [27] give values of $6.8 \mathrm{emu} / \mathrm{g}\left(M_{s}\right), 1025 \mathrm{Oe}\left(H_{c}\right)$, and 0.27 remanence ratio $(R)$ at room temperature, whereas $13-\mathrm{nm}$ cobalt ferrite nanoparticles made by the polymeric precursor method [11] give $67.5 \mathrm{emu} / \mathrm{g}\left(M_{s}\right), 1842 \mathrm{Oe}\left(H_{c}\right)$, and 0.5 remanence ratio for similar conditions.

\section{Conclusion}

We have succeeded in synthesizing spinel cobalt ferrite $\left(\mathrm{CoFe}_{2} \mathrm{O}_{4}\right)$ nanoparticles by a thermal treatment method utilizing only cobalt nitrate and iron nitrate as precursors, PVP as an agglomeration capping agent, and deionized water as a solvent. Particle sizes of 12.5-39 nm were obtained with calcination temperatures between 623 and $923 \mathrm{~K}$, as confirmed by TEM and XRD analyses. Calcination at 823 and $923 \mathrm{~K}$ completely removed the organic compounds and nitrate ions. The magnetic studies showed that the saturation, magnetization, and remanent magnetization of the cobalt ferrite nanoparticles increased as temperature increased while the coercivity field and remanence ratio increased, until they achieved a maximum value and then decreased. This simple, cost-effective, and environmentally friendly method that produces no by-product effluents can be used to synthesize pure crystalline spinel cobalt ferrite nanoparticles. Furthermore, it can be extended to synthesizing other spinel ferrite nanoparticles of interest in nanotechnology.

\section{Acknowledgments}

The authors would like to thank Miss. Fatemeh Moazami Goodarzi for her great assistance in the chemical mechanism study. This work was supported by the Ministry of Higher Education of Malaysia under the FRGS grant and Universiti Putra Malaysia under the RUGS grant.

\section{References}

[1] B. Aslibeiki, P. Kameli, H. Salamati, M. Eshraghi, and T. Tahmasebi, "Superspin glass state in $\mathrm{MnFe}_{2} \mathrm{O}_{4}$ nanoparticles," Journal of Magnetism and Magnetic Materials, vol. 322, no. 19, pp. 2929-2934, 2010.

[2] A. P. Alivisatos, "Semiconductor clusters, nanocrystals, and quantum dots," Science, vol. 271, no. 5251, pp. 933-937, 1996.

[3] M. Sugimoto, "The past, present, and future of ferrites," Journal of the American Ceramic Society, vol. 82, no. 2, pp. 269280, 1999.

[4] H.-M. Fan, J.-B. Yi, Y. Yang et al., "Single-crystalline $\mathrm{MFe}_{2} \mathrm{O}_{4}$ nanotubes/nanorings synthesized by thermal transformation process for biological applications," ACS Nano, vol. 3, no. 9, pp. 2798-2808, 2009.

[5] M. H. Khedr, A. A. Omar, and S. A. Abdel-Moaty, "Magnetic nanocomposites: preparation and characterization of Coferrite nanoparticles," Colloids and Surfaces A, vol. 281, no. 13, pp. 8-14, 2006.

[6] J. B. Silva, W. De Brito, and N. D. S. Mohallem, "Influence of heat treatment on cobalt ferrite ceramic powders," Materials Science and Engineering B, vol. 112, no. 2-3, pp. 182-187, 2004.

[7] Z. Zi, Y. Sun, X. Zhu, Z. Yang, J. Dai, and W. Song, "Synthesis and magnetic properties of $\mathrm{CoFe}_{2} \mathrm{O}_{4}$ ferrite nanoparticles," Journal of Magnetism and Magnetic Materials, vol. 321, no. 9, pp. 1251-1255, 2009.

[8] V. L. Calero-DdelC and C. Rinaldi, "Synthesis and magnetic characterization of cobalt-substituted ferrite $\left(\mathrm{Co}_{x} \mathrm{Fe}_{3-x} \mathrm{O}_{4}\right)$ nanoparticles," Journal of Magnetism and Magnetic Materials, vol. 314, no. 1, pp. 60-67, 2007.

[9] D. Zhao, X. Wu, H. Guan, and E. Han, "Study on supercritical hydrothermal synthesis of $\mathrm{CoFe}_{2} \mathrm{O}_{4}$ nanoparticles," Journal of Supercritical Fluids, vol. 42, no. 2, pp. 226-233, 2007.

[10] L. Chen, Y. Shen, and J. Bai, "Large-scale synthesis of uniform spinel ferrite nanoparticles from hydrothermal decomposition of trinuclear heterometallic oxo-centered acetate clusters," Materials Letters, vol. 63, no. 12, pp. 1099-1101, 2009. 
[11] M. Gharagozlou, "Synthesis, characterization and influence of calcination temperature on magnetic properties of nanocrystalline spinel Co-ferrite prepared by polymeric precursor method," Journal of Alloys and Compounds, vol. 486, no. 1-2, pp. 660-665, 2009.

[12] I. H. Gul and A. Maqsood, "Structural, magnetic and electrical properties of cobalt ferrites prepared by the sol-gel route," Journal of Alloys and Compounds, vol. 465, no. 1-2, pp. 227231, 2008.

[13] V. Pillai and D. O. Shah, "Synthesis of high-coercivity cobalt ferrite particles using water-in-oil microemulsions," Journal of Magnetism and Magnetic Materials, vol. 163, no. 1-2, pp. 243248, 1996.

[14] J. Zhang and C. Q. Lan, "Laser-generated Ni and Co nanoparticles in organic media," Materials Letters, vol. 62, no. 10-11, pp. 1521-1524, 2008.

[15] G. Baldi, D. Bonacchi, C. Innocenti, G. Lorenzi, and C. Sangregorio, "Cobalt ferrite nanoparticles: the control of the particle size and surface state and their effects on magnetic properties," Journal of Magnetism and Magnetic Materials, vol. 311, no. 1, pp. 10-16, 2007.

[16] K. V. P. M. Shafi, A. Gedanken, R. Prozorov, and J. Balogh, "Sonochemical preparation and size-dependent properties of nanostructured $\mathrm{CoFe}_{2} \mathrm{O}_{4}$ particles," Chemistry of Materials, vol. 10, no. 11, pp. 3445-3450, 1998.

[17] S. Singhal, J. Singh, S. K. Barthwal, and K. Chandra, "Preparation and characterization of nanosize nickel-substituted cobalt ferrites $\left(\mathrm{Co}_{1-x} \mathrm{Ni}_{x} \mathrm{Fe}_{2} \mathrm{O}_{4}\right)$," Journal of Solid State Chemistry, vol. 178, no. 10, pp. 3183-3189, 2005.

[18] J. Jiang and L. H. Ai, "Synthesis and characterization of FeCo binary ferrospinel nanospheres via one-step nonaqueous solution pathway," Materials Letters, vol. 64, no. 8, pp. 945947, 2010.

[19] Y. Sui, X. Huang, Z. Ma et al., "The effect of thermal annealing on crystallization in a- $\mathrm{Si}: \mathrm{H} / \mathrm{SiO}_{2}$ multilayers by using layer by layer plasma oxidation," Journal of Physics Condensed Matter, vol. 15, no. 34, pp. 5793-5800, 2003.

[20] P. Laokul, V. Amornkitbamrung, S. Seraphin, and S. Maensiri, "Characterization and magnetic properties of nanocrystalline $\mathrm{CuFe}_{2} \mathrm{O}_{4}, \mathrm{NiFe}_{2} \mathrm{O}_{4}, \mathrm{ZnFe}_{2} \mathrm{O}_{4}$ powders prepared by the Aloe vera extract solution," Current Applied Physics, vol. 11, no. 1, pp. 101-108, 2011.

[21] M. I. Mendelson, "Average grain size in polycrystalline ceramics," Journal of American Ceramic Society, vol. 52, no. 8, pp. 443-446, 1969.

[22] B. D. Cullity, Elements of X-Ray Diffraction, Addison-Wesley, London, UK, 2nd edition, 1978.

[23] M. I. Loría-Bastarrachea, W. Herrera-Kao, J. V. CauichRodríguez, J. M. Cervantes-Uc, H. Vázquez-Torres, and A. Ávila-Ortega, "A TG/FTIR study on the thermal degradation of poly(vinyl pyrrolidone)," Journal of Thermal Analysis and Calorimetry. In press.

[24] M. Sangmanee and S. Maensiri, "Nanostructures and magnetic properties of cobalt ferrite $\left(\mathrm{CoFe}_{2} \mathrm{O}_{4}\right)$ fabricated by electrospinning," Applied Physics A, vol. 97, no. 1, pp. 167-177, 2009.

[25] V. A. M. Brabers, "Infrared spectra of cubic and tetragonal manganese ferrites," Physica Status Solidi, vol. 33, no. 2, pp. 563-572, 1969.

[26] Y. J. Kwon, K. H. Kim, C. S. Lim, and K. B. Shim, "Characterization of $\mathrm{ZnO}$ nanopowders synthesized by the polymerized complex method via an organochemical route," Journal of Ceramic Processing Research, vol. 3, no. 3, pp. 146-149, 2002.
[27] Y. Qu, H. Yang, N. Yang, Y. Fan, H. Zhu, and G. Zou, "The effect of reaction temperature on the particle size, structure and magnetic properties of coprecipitated $\mathrm{CoFe}_{2} \mathrm{O}_{4}$ nanoparticles," Materials Letters, vol. 60, no. 29-30, pp. 35483552, 2006.

[28] M. K. Roy, B. Haldar, and H. C. Verma, "Characteristic length scales of nanosize zinc ferrite," Nanotechnology, vol. 17, no. 1, pp. 232-237, 2006.

[29] T. Giannakopoulou, L. Kompotiatis, A. Kontogeorgakos, and G. Kordas, "Microwave behavior of ferrites prepared via solgel method," Journal of Magnetism and Magnetic Materials, vol. 246, no. 3, pp. 360-365, 2002.

[30] M. P. Gonzalez-Sandoval, A. M. Beesley, M. Miki-Yoshida, L. Fuentes-Cobas, and J. A. Matutes-Aquino, "Comparative study of the microstructural and magnetic properties of spinel ferrites obtained by co-precipitation," Journal of Alloys and Compounds, vol. 369, no. 1-2, pp. 190-194, 2004.

[31] F. Nakagomi, S. W. Da Silva, V. K. Garg et al., "The influence of cobalt population on the structural properties of $\mathrm{Co}_{x} \mathrm{Fe}_{3-x} \mathrm{O}_{4}$," Journal of Applied Physics, vol. 101, no. 9, pp. 09M51409M517, 2007.

[32] E. V. Gopalan, I. A. Al-Omari, D. S. Kumar, Y. Yoshida, P. A. Joy, and M. R. Anantharaman, "Inverse magnetocaloric effect in sol-gel derived nanosized cobalt ferrite," Applied Physics A, vol. 99, no. 2, pp. 497-503, 2010.

[33] A. Franco Jr. and F. C. E Silva, "High temperature magnetic properties of cobalt ferrite nanoparticles," Applied Physics Letters, vol. 96, no. 17, pp. 172505-172508, 2010.

[34] Y. Kinemuchi, K. Ishizaka, H. Suematsu, W. Jiang, and K. Yatsui, "Magnetic properties of nanosize $\mathrm{NiFe}_{2} \mathrm{O}_{4}$ particles synthesized by pulsed wire discharge," Thin Solid Films, vol. 407, no. 1-2, pp. 109-113, 2002.

[35] R. H. Kodama, A. E. Berkowitz, E. J. McNiff, and S. Foner, "Surface spin disorder in ferrite nanoparticles," Journal of Applied Physics, vol. 81, no. 8, pp. 5552-5557, 1997.

[36] D. S. Mathew and R. S. Juang, "An overview of the structure and magnetism of spinel ferrite nanoparticles and their synthesis in microemulsions," Chemical Engineering Journal, vol. 129, no. 1-3, pp. 51-65, 2007.

[37] Z. L. Wang, Y. Liu, and Z. Zhang, Handbook of Nanophase and Nanostructured Materials. Materials Systems and Applications I, Vol. III, Kluwer Academic, Boston, Mass, USA; Plenum, New York, NY, USA, 2003.

[38] E. C. Stoner and E. P. Wohlfarth, "A mechanism of magnetic hysteresis in heterogeneous alloys," Philosophical Transactions of the Royal Society of London, vol. 240, pp. 599-642, 1948.

[39] M. Vasic, B. Antic, A. Kremenovic et al., "Zn,Ni ferrite/NiO nanocomposite powder obtained from acetylacetonato complexes," Nanotechnology, vol. 17, no. 19, pp. 4877-4884, 2006.

[40] M. George, A. Mary John, S. S. Nair, P. A. Joy, and M. R. Anantharaman, "Finite size effects on the structural and magnetic properties of sol-gel synthesized $\mathrm{NiFe}_{2} \mathrm{O}_{4}$ powders," Journal of Magnetism and Magnetic Materials, vol. 302, no. 1, pp. 190-195, 2006.

[41] B. D. Cullity, Introduction to Magnetic Materials, AddisonWesley, Reading, Mass, USA, 1972.

[42] S. Chikazumi, Physics of Magnetism, Wiley, New York, NY, USA, 1959. 

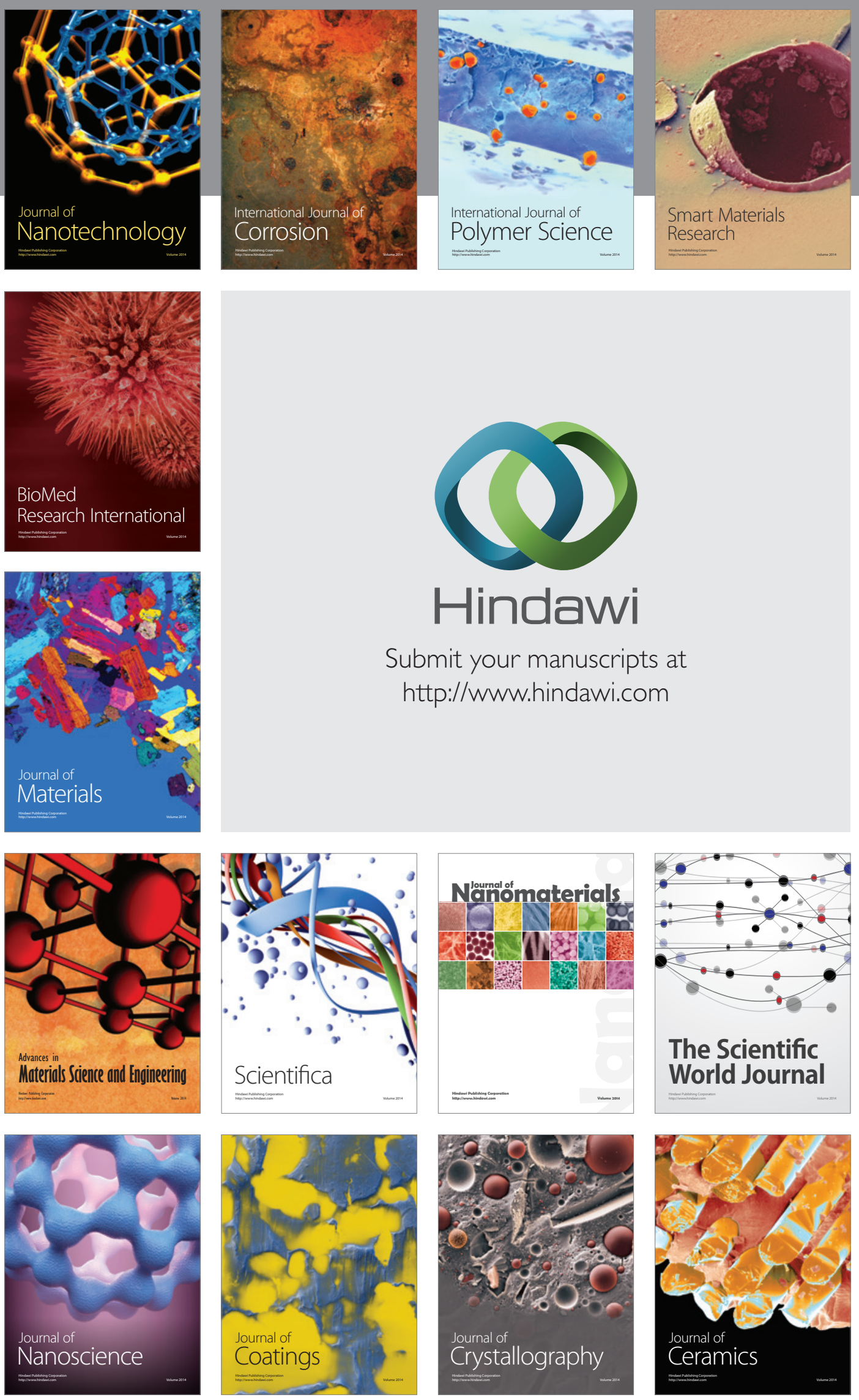

The Scientific World Journal

Submit your manuscripts at

http://www.hindawi.com

\section{World Journal}

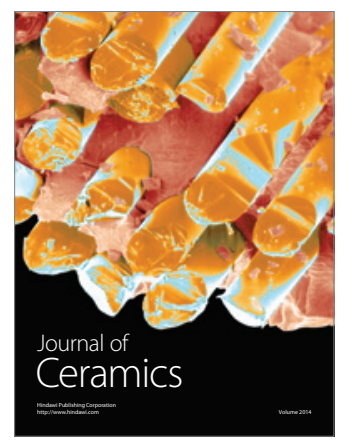

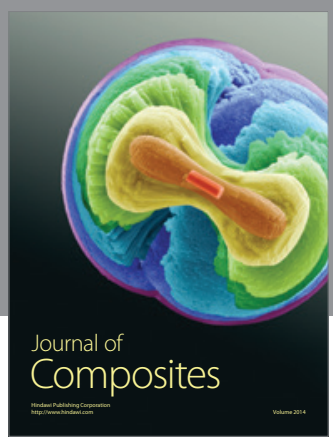
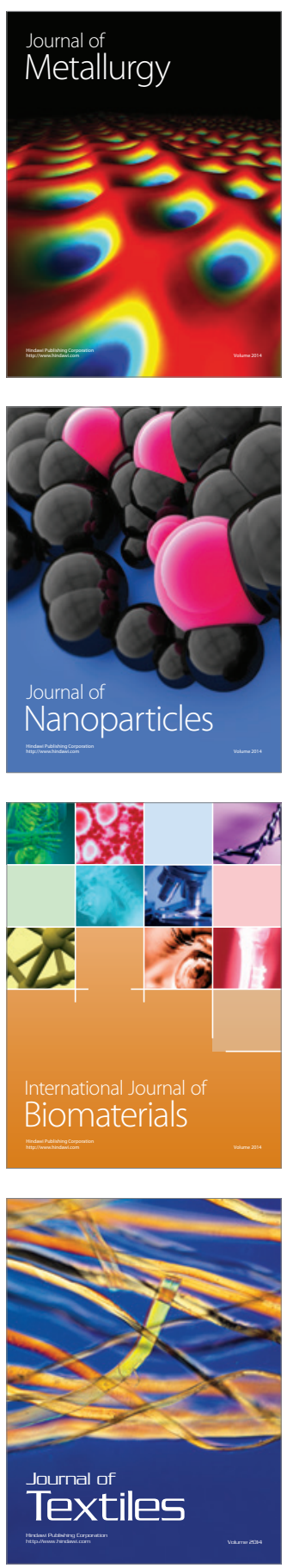